\title{
El cuerpo de la experiencia estética
}

\author{
Hernán Javier Pinzón Manrique ${ }^{1}$
}

\section{Resumen}

Este artículo plantea un problema dentro de la concepción de la experiencia estética en el mundo occidental: la implicación sobre la escisión entre cuerpo y mente. Así, se busca presentar una aproximación a algunos postulados significativos que propone el mundo contemporáneo donde se reconoce la experiencia del sujeto como cuerpo en el mundo. En estos términos, la estética se propone como una experiencia humana de exploración del sujeto en su cotidianidad y singularidad, que parte de la relación entre la experiencia encarnada y el mundo de la vida. Por tanto, la estética no se reduce a una teoría sobre el arte y la belleza, sino que se amplía a las formas de significación desde la percepción del cuerpo vivido.

Palabras clave: conciencia encarnada, cuerpo, experiencia estética, fenomenología del cuerpo, percepción, posestructuralismo. 


\title{
The body of the aesthetic experience
}

\begin{abstract}
This article sets out a problem inside the esthetic experience conception in the western world: the involvement about the Split between body and mind. In that way, it is sought to present closeness to some significant postulates that propose the contemporary world where it recognizes the subject experience as the body in the world. In these terms, esthetics is proposed as a human experience of the subject exploration in its daily activities and singularity that comes of the relation between the embodied experience and the life world. Therefore, esthetics does not reduce to a theory about art and beauty, but rather increases to the forms of significance since the living body perception.
\end{abstract}

Keywords: embodied conscience, body, esthetic experience, body phenomenology, perception, post-structuralism.

Recibido: 18/08/2014

Aceptado: 23/10/2014

\section{Introducción}

Un punto de partida hacia la comprensión y superación sobre el panorama de escisión entre cuerpo y mente en el mundo contemporáneo, es la experiencia del personaje principal de la novela de Thomas Mann La montaña mágica (1997). Dicha experiencia se puede definir como el develamiento que sufre Hans Castorp cuando observa su mano, y que constituye un signo sobre el reconocimiento de su carnalidad, de su fragilidad, de cómo la muerte trastoca el amplio horizonte de la experiencia humana.

El paulatino hecho de reconocerse como carne implica en el personaje de la obra la apertura a la existencia humana como cuerpo, y que constituye el punto de partida de su nueva experiencia con el mundo. En este marco ¿Qué es el cuerpo como experiencia? y ¿Qué implicaciones tiene como punto de partida de la experiencia estética? Estos son interrogantes que parten de la develación sobre la experiencia del cuerpo en un personaje literario que reconoce su inevitable fragilidad, y que asume el horizonte culminante de la experiencia humana en dicho ámbito: la muerte. 
Y Hans Castorp vio lo que debía de haber esperado, pero que en suma no está hecho para ser visto por el hombre, y que nunca hubiera creído que pudiera ver: miró dentro de su propia tumba. Vio el futuro trabajo de la descomposición, lo vio prefigurado por la fuerza de la luz, vio la carne, en la que él vivía, descompuesta, aniquilada, disuelta en una niebla inexistente, y en medio de ella el esqueleto, cincelado esmeradamente, de su mano derecha, en torno de cuyo anular la sortija de su abuelo flotaba negra y fea: un objeto duro y telúrico con el que el hombre adorna su cuerpo destinado a desaparecer, de modo que, una vez libre, vaya hacia otra carne que podrá lucirlo un nuevo lapso de tiempo con los ojos de la abuela Tienappel, veía un miembro familiar de su cuerpo, eran ojos penetrantes de visionario, y por primera vez en su vida comprendió que estaba destinado a morir (Mann, 1997: 301-302).

Este fragmento de la obra literaria presenta el momento en que Hans Castorp se hace consciente de su habitud en la carne, y sobre el destino fatal de dicha experiencia en la corrupción del cuerpo humano. Este evento, que sucede mientras es sometido a un examen de rutina en el sanatorio de Berghof, permite a partir del reconocimiento de la enfermedad, otra forma de experimentar la vida por fuera de los valores del mundo que acaece más allá de la montaña: el progreso y el orden, la racionalidad, el campo de producción, es decir, el mundo moderno que no hace parte de la forma de vida de los habitantes del sanatorio.

\section{Metodología}

Desde el texto de La Montaña Mágica de Thomas Mann (1997), se propone una interpretación sobre el significado del cuerpo como representación en el mundo contemporáneo, que replantea la visión humana de la escisión con la mente. Así, desde la propuesta fenomenológica de la percepción de Maurice MerleauPonty, se busca un acercamiento a la definición de cuerpo como experiencia fundamental del ser humano con el mundo de la vida.

En este marco, se hace un rastreo de las ideas fundamentales en la historia del pensamiento occidental sobre la estética, encontrando en el planteamiento de Michel Foucault, una perspectiva que permite comprender una nueva categorización sobre lo estético desde el sujeto definido como cuerpo. Se toma, en este marco, como punto de partida de la experiencia estética la noción de conciencia encarnada, referenciada en la narración que presenta Virginia Woolf en su novela Las Olas (1980), donde el cuerpo constituye el punto de partida sobre una experiencia con el mundo y con el lenguaje. 


\section{Hacia una definición del cuerpo desde la percepción}

La experiencia de Castorp es fundamento de la experiencia humana en el mundo a partir del cuerpo, y donde la escisión con la mente se supera desde la incorporación de toda actividad humana: «How are body and mind done, not two? frames our whole conception of the relation dualistically, since it presupposes that two different kinds of things must somehow come together into one» (Johnson, 2007: 7). Este planteamiento de Mark Johnson determina un panorama central sobre la dualidad presente dentro de la concepción humana en Occidente. La síntesis de esta dualidad histórica, y como antecedente fundamental para las teorías sobre la incorporación, es abordada en el siglo XX por el pensador francés Maurice Merleau-Ponty (1908-1961), como heredero del pensamiento fenomenológico.

Una de sus obras fundamentales Fenomenología de la percepción (1984), presenta al cuerpo humano como el órgano de conexión con el mundo de la vida (lebenswelt), desde una experiencia definida como percepción que posibilita una interconexión que tienen su esencia en la ambigüedad, en la imperfección al ser finita, inacabada y precaria (Descombes, 1982).

En estos términos ¿Cuál es el significado de la percepción como síntesis fenomenológica de la escisión entre cuerpo y mente? Para responder a este interrogante, acerquémonos al propósito de un nuevo panorama de la fenomenología propuesto por Merleau-Ponty dentro de su investigación del mundo humano:

la fenomenología sería en definitiva el proyecto de una descripción del fundamento de la historia, a saber, la existencia humana, tal como es vivida, es decir, nunca completamente blanca o negra, sino abigarrada, mezclada. Esta mixtura es lo que hay que describir: un producto-productor, un activo-pasivo, un instituido-instituyente, es decir, en todas estas figuras, un sujeto-objeto (Descombes, 1982: 83).

En estos términos, la percepción desde la postura merleaupontyana es la inherencia al mundo que provee un conocimiento originario a partir de una concepción encarnada de la conciencia. Dicha experiencia perceptiva es la compresión sobre la inserción o penetración recíprocas de los fenómenos, definida como intraontología al plantear una «inserción recíproca» o «complicidad promiscua» en la experiencia (Bech, 2005: 15).

Sobre este punto es importante anotar que la promiscuidad, que etimológicamente se relaciona con el alcance de la mixtura, se puede interpretar como la experiencia humana incorporada, donde el sujeto y el mundo constituyen la génesis del sentido, frente a un cogito que busca la abstracción, la pureza, y que aleja a la racionalidad del enturbiamiento de la experiencia con el mundo. 
La percepción, como fenómeno incorporado, es el ámbito donde los sentidos se conectan, y que implica una noción amplia sobre las concepciones de pensamiento y conocimiento que en la tradición moderna corresponden a la razón y la objetividad. Por tanto, la conciencia reflexiva, la aprehensión del mundo por medio de la idea no posibilita el fundamento de la experiencia, sino la conciencia corporal que abarca el conocer, sentir, pensar, imaginar (MerleauPonty, 1984).

Retomemos la experiencia de Castorp dentro del sanatorio, y establezcamos dentro de su advenimiento de la carne, una construcción de sentido. Antes de esta experiencia se podría determinar que Castorp estaba limitado en su relación con el mundo desde los valores del campo de producción, del mundo moderno. Su revelación es significativa al descifrar y crear posibilidades de construcción de sentido desde la subjetividad, desde la exploración de sí mismo. Por tanto, es fundamental una aproximación al cómo la experiencia perceptiva posibilita la construcción sobre dicho proceso, y que se relaciona con el campo de la estética.

\section{Una aproximación a la escisión de la experiencia estética}

Partamos de una definición del fenómeno estético como una investigación sobre mi construcción de sentido. La estética es definida por Mark Johnson como «properly an investigation of everything that goes into the human meaningmaking, and its traditional focus on the arts stems primarily from the fact that arts are exemplary cases of consummated meaning» (2007: preface, XI). Más que una teoría estética, es fundamental plantear una experiencia que explore cómo el significado humano es posible desde la relación de nuestro cuerpo con el mundo de la vida.

Sin embargo, dicha construcción humana del significado tiene un proceso histórico que permite la pregunta por ¿Qué es la experiencia estética?, ¿Cómo se relaciona con el cuerpo? Este interrogante se aproxima a las implicaciones de la dualidad mente-cuerpo dentro de dicho campo, y cómo el proceso de incorporación dentro del mundo contemporáneo le permite un horizonte de investigación que supera su histórico objeto de estudio: el arte y la belleza. Por tanto, no se debe considerar a la Estética como una teoría consistente y unificada en su discurso sobre el tiempo. Foucault (1970), propone una perspectiva sobre la historia y los enunciados que la componen, que se puede articular con la necesidad de encontrar un replanteamiento sobre la concepción de la experiencia estética:

¿Qué son pues, la medicina, la gramática, la economía, la política?, ¿No son nada, sino una reagrupación retrospectiva por la cual las ciencias contemporáneas se hacen una ilusión en cuanto a su propio pasado?, ¿Son formas que se han instaurado de una vez para siempre y se han 
desarrollado soberanamente a través del tiempo?, ¿Cubren otras unidades?, ¿Y qué especie de relaciones hemos de reconocer valederas entre todos esos enunciados que forman, sobre un modo a la vez familiar e insistente, una masa enigmática? (Foucault, 1970: 51).

Desde esta perspectiva, Foucault invita a pensar en la imposibilidad sobre la unidad en la caracterización de un enunciado, hay una ruptura entre los enunciados, una multiplicidad que no permite determinarlo como un objeto único de algo. En este caso lo importante, lo que constituye el horizonte del trabajo es por un lado la escisión cuerpo-mente en la experiencia estética, por otro, la ruptura con la idealidad de dicha experiencia y su aterrizaje en el contexto de la cotidianidad.

Una definición tentativa de la estética puede partir del origen del concepto a mediados del siglo XVIII. Anterior a esta designación se puede encontrar en la historia de las ideas filosóficas, la pregunta por el arte, sus valores y posibilidades en términos de conocimiento. El punto de partida de la escisión se encuentra para Occidente en el mundo griego, que estaba determinado por una perspectiva metafísica que se imponía sobre la validez de la experiencia sensible.

Este es el caso de Platón con la idea de lo bello y la condena sobre el papel del arte dentro de la República, además de la subordinación de la belleza a la idea de bien, lo que constituye un predominio de lo epistemológico y ético sobre lo estético. En Aristóteles, que reivindica un punto de partida sobre lo sensorial, establece una relación clave con el tema de la primacía racional, donde la belleza constituye un estímulo en relación con la physis. Es importante resaltar, dentro de este marco de pensamiento, que el artista no es creador, sino que constituye un realizador de la representación en términos de la mímesis (Ocampo, 2003).

El neoplatonismo y el neoaristotelismo que se desarrolló desde una perspectiva cristiana con Agustín de Hipona y Tomás de Aquino, discute las mismas categorizaciones metafísicas dentro de su designación con los términos de la teología cristiana. Lo fundamental dentro de estos dos momentos de la escisión entre cuerpo y mente, es la primacía de lo racional, lo espiritual y los valores objetivos dentro de la posibilidad de la experiencia estética.

Sin embargo, una ruptura importante dentro de la dicotomía en cuestión es el planteamiento estético del renacimiento con la idea de sujeto y cotidianidad en relación con la idea de belleza, que significa una tentativa de ruptura con el teocentrismo.

Con Alexander Baumgarten (1714-1762), se establece una distinción del plano estético en relación con el carácter sentimental de lo bello, la belleza como un sentimiento, y la imposibilidad de lo racional en la consideración sobre lo estético. Con Kant se puede determinar una psicología de la sensibilidad a partir de centrar su interés en el juicio estético de lo sublime que genera la contemplación de la 
belleza, y cómo desde este juicio subjetivo se puede establecer la posibilidad de validez; sin embargo, el filósofo alemán mantiene una ruptura en lo espiritual y material del plano estético. Con Hegel se conserva la misma escisión donde la materialización del espíritu en el arte constituye el punto de partida de su estética.

En el siglo XX las vanguardias cumplen un papel fundamental al poner en crisis el sentido clásico y moderno de la estética y del arte. El punto de partida de esta crisis es el cuestionamiento de la normatividad en el arte, lo que constituye una paradoja frente a su nueva naturaleza.

Esta aproximación histórica permite comprender la ruptura fundamental en el pensamiento estético desde la escisión cuerpo-mente dentro de la experiencia estética. Así mismo, posibilita comprender dos dimensiones dentro del arte: una en cuanto a la representación y la otra en cuanto a la imagen del artista como creador (Cárdenas, 2004: 12). La primera enmarcada en una serie de valores preestablecidos como la belleza, mientras que la segunda presenta una serie de valores que son el resultado de una construcción subjetiva por parte del artista.

La estética posestructuralista enmarcada en la filosofía francesa del siglo $\mathrm{XX}$, presenta una concepción de la estética en relación con el análisis de la subjetividad, de lo cotidiano y la singularidad que amplían el hecho estético del abordaje de textos artísticos. En este marco, la subjetividad es entendida como formas de vida, de expresión que se concentran en una práctica, y la estética como un saber que busca comprender a la gente en sus prácticas del cuerpo. Dentro del marco del posestructuralismo, el planteamiento del pensador francés Michel Foucault es importante para comprender el nuevo sentido del concepto de estética dentro del mundo contemporáneo. El punto de partida para comprender su concepción está en la construcción sobre la idea de sujeto.

En el texto Tecnologías del yo (Foucault, 1990), parte de la pregunta por ¿Cómo se obligó al sujeto a descifrarse a sí mismo a partir de lo que estaba prohibido? (Foucault, 1990: 47), como punto de partida para configurar una noción de sujeto. Foucault hace un rastreo de las formas en que la cultura y los hombres han desarrollado un saber de sí mismos, buscando el desarrollo de una hermenéutica de las tecnologías del yo en las costumbres del paganismo y del cristianismo primitivo, definiendo dicho tipo de tecnologías como las que «permiten a los individuos efectuar, por cuenta propia o con la ayuda de otros, cierto número de operaciones sobre su cuerpo y su alma, pensamientos, conducta, o cualquier forma de ser, obteniendo así una transformación de sí mismos con el fin de alcanzar cierto estado de felicidad, pureza, sabiduría o inmortalidad» (Foucault, 1990: 48).

En estos términos, se llega al concepto grecorromano de Epimelesthai Santoi, entendido como el cuidado de sí, la preocupación de si, el sentirse preocupado, inquieto por sí. La idea sobre el cuidado de sí se diferencia de otro concepto 
sobre la subjetividad en el contexto clásico a partir de la idea conócete a ti mismo, que implica una perspectiva más cartesiana centrada en el pensamiento, mientras que la primera apunta a las prácticas que constituyen la preocupación por el cuerpo (Foucault, 1990: 65).

Este planteamiento busca proponer la superación de la experiencia con el mundo a partir del cogito, para constituirse dentro de la experiencia humana como una conciencia encarnada, y tiene un referente importante dentro de la novela de la escritora Virginia Woolf Las olas (1980).

\section{La experiencia estética desde la conciencia encarnada}

En La montaña mágica (1997), encontramos la idea de la corporalidad como proceso al que llega Hans Castorp como experiencia, donde aparece el cuerpo como representación, como posible tema dentro de la narración. Esta es una perspectiva para referirnos al cuerpo. Paralela a este punto, podremos encontrar el caso de la novela Las olas (1980), de Virginia Wolf, como ejemplo de la literatura que parte del cuerpo en relación con el lenguaje, y que proporciona la experiencia perceptiva de la conciencia encarnada, a partir de las voces de los personajes presentes en la narración (Bernard, Neville, Louis, Susan, Horda y Jiny).

Cada voz constituye un sujeto sintiente-sensible, en términos de Merleau-Ponty (1984), desde la experiencia perceptiva de afectación con el mundo. A partir de la palabra como generadora de significado, se posibilita entablar la comprensión singular sobre el mundo, como experiencia perceptiva que adquiere dimensiones estéticas:

ahora comienza a alzarse en mí el conocido ritmo. Palabras que yacían dormidas se alzan ahora, agitan la cresta, suben y bajan, vuelven a subir y a bajar. Soy un poeta, sí, lo soy. Y seguramente soy un gran poeta. Barcas y muchachos que pasan...Lo veo todo, lo siento todo. Estoy inspirado. De mis ojos rebosan las lágrimas. Pero, mientras experimento estas sensaciones, estimulo a latigazos mi frenesí para que suba más y más. Espumea. Deviene artificial e insincero. Palabras, palabras y palabras, cómo galopan (Woolf, 1980: 77).

El entretejido narrativo está constituido por una afluencia de impresiones sensibles que los personajes alternadamente van delineando y constituyendo en una descripción de imágenes, sonidos, texturas, sensaciones "El agua me recorre la espina dorsal. Destellantes flechas de sensaciones se disparan hacia uno y otro lado. Estoy cubierto de cálida carne. Las secas coyunturas se me humedecen; mi cuerpo frío se calienta, chorrea y brilla...» (Woolf, 1980: 22). La efusión de percepciones corporales sobre el mundo y el constante reconocerse de los personajes imprimen significado en el acontecer de sus vivencias «Ahora, 
especialmente ahora, que acabo de abandonar una estancia, con gente hablando en ella, y que las losas resuenan a mi paso solitario, y que miro a la luna alzándose sublime e indiferente sobre la antigua capilla, ahora veo con gran claridad que no soy uno y simple, sino múltiple y complejo» (Woolf, 1980: 71).

Otra característica de la conciencia encarnada presente en la novela es la correlación de facultades implicadas en la percepción, trascendiendo la oposición del mundo interno y externo, de la razón y el sentimiento, del entendimiento y la intuición "“junto a una sensibilidad de mujer" (palabras de mi biógrafo) 'Bernard estaba dotado del rigor lógico masculino'... Entre vosotros, que ahora estáis hablando de mí, pocos son los que tienen la doble capacidad de sentir y razonar» (Woolf, 1980: 72). Las olas constituye la comprensión del devenir de la vida, del tiempo, de la existencia en la carne como postura y síntesis del problema de la dualidad desde la percepción sublime sobre «la visión de un momento» (Woolf, 1980: 61). Esta perspectiva sugiere una concepción de la experiencia como una forma de ser- en- el-mundo desde la multidimensional de la experiencia humana en relación con el propio cuerpo, el mundo de la vida y los otros, constituyendo un contacto que no solamente tiene que ver con el pensamiento, sino que se amplía a una imbricación desde lo sensible. A este tipo de interconexión, que genera unos procesos de significación, se le puede definir como una estética de la percepción.

\section{Conclusiones}

El problema de la dualidad como estructura que sostiene la idea de conocimiento en Occidente, presenta sus implicaciones dentro de la concepción sobre la estética y el arte. La percepción constituye un acto fundamental dentro del ser humano, sin embargo, y partiendo de la idea sobre la percepción singular que tiene el artista, se encuentra una conexión con la teoría estética en cuanto presenta una forma particular de contemplación-comprensión de la realidad. A pesar de esto, el problema de la dualidad es implícito a la definición de la experiencia estética dentro de una dimensión intelectual que no parte de una relación significativa con el mundo de la vida, aunque, desde la perspectiva de Merleau-Ponty se replantea la experiencia del cuerpo y la posibilidad de sentido.

La experiencia estética surge de la percepción sobre el mundo, su alcance trasciende el discurso sobre la observación de los fenómenos artísticos para constituirse en una forma de relación humana con la realidad y con la posibilidad de construir sentidos. El pensador norteamericano John Dewey (1859-1952), en su texto El arte como experiencia (2008), plantea que el entendimiento sobre lo estético y sobre el arte se basa en la materia prima de acontecimientos y escenas sobre los sentidos, despertando el interés y el goce en la contemplación (2008: 5). Esto implica un punto de partida desde la experiencia del cuerpo como integración de las múltiples facultades humanas. 
El rescate del sujeto conlleva una identificación con la experiencia del cuerpo, desde la superación de la dualidad por medio de la unión estructural desde la conciencia en el cuerpo y sus posibilidades perceptivas. Por tanto, el pensamiento, el sentimiento, son corpóreos desde una experiencia estética de la percepción. En estos términos, la posibilidad de dar sentido sobre dicha experiencia determina la singularidad del sujeto y la configuración de su mundo desde el lenguaje.

\section{Referencias bibliográficas}

Beardsley, M. \& Hospers, J. 1981. Estética, historia y fundamentos. Madrid: Ediciones Cátedra.

Bech, J. 2005. Merleau-Ponty: Una aproximación a su pensamiento. Barcelona: Anthropos Editorial.

Cárdenas, A. 2004. Elementos para una pedagogía de la literatura. Bogotá: Universidad Pedagógica Nacional.

Descombes, V. 1982. Lo mismo y lo otro: cuarenta y cinco años de filosofía francesa (1933-1978). Madrid: Ediciones Cátedra.

Dewey, J. 2008. El arte como experiencia. Barcelona: Paidós.

Foucault, M. 1990. Tecnologías del yo y otros textos afines. Barcelona: Paidós.

Foucault, M. 1970. La arqueología del saber. Buenos Aires: Siglo XXI Editores.

Johnson, M. 2007. The Meaning of the Body: Aesthetics of Human Understanding. Chicago: University of Chicago Press.

Mann, T. 1997. La montaña mágica. Barcelona: Edhasa.

Merleau-Ponty, M. 1984. Fenomenología de la percepción. Barcelona: Editorial PlanetaDe Agostini.

Ocampo, E. 2003. Estética de la naturaleza, del arte, de las artesanías. En: Enciclopedia Iberoamericana de filosofía v. 25: 23-45. Estética. Madrid: Editorial Trotta.

Woolf, V. 1980. Las Olas. Barcelona: Bruguera. 